\author{
P. C. Sundgren \\ Q. Dong \\ D. Gómez-Hassan \\ S. K. Mukherji \\ P. Maly \\ R. Welsh
}

\section{Diffusion tensor imaging of the brain: review of clinical applications}

Received: 15 July 2003

Accepted: 2 September 2003

Published online: 21 April 2004

(C) Springer-Verlag 2004

P. C. Sundgren $(\bowtie) \cdot$ Q. Dong

D. Gómez-Hassan · S. K. Mukherji

P. Maly $\cdot$ R. Welsh

Department of Radiology, University of

Michigan Health System, 1500 E. Medical

Center Drive, Ann Arbor, MI 48109-0030,

USA

E-mail: sundgren@umich.edu

Tel.: + 1-734-7633253

Fax: + 1-734-7642142

\begin{abstract}
We review the theoretical background to diffusion tensor imaging (DTI) and some of its commoner clinical applications, such as cerebral ischemia, brain maturation and traumatic brain injury. We also review its potential use in diseases such as epilepsy, multiple sclerosis, and Alzheimer's disease. The value of DTI in the investigation of brain tumors and metabolic disorders is assessed.
\end{abstract}

Keywords Magnetic resonance imaging $\cdot$ Diffusion tensor imaging $\cdot$ Brain

\section{Background}

Diffusion-weighted imaging (DWI) is a well-established method implemented as a part of a routine protocol at many institutions. It shows the diffusivity of water molecules [1] and the principles of measurement of diffusion with MRI are well described $[2,3]$. DWI has shown to be very useful for early signs of ischemia $[4,5]$, but is also increasingly used in the investigation of other brain diseases, e.g., multiple sclerosis $[6,7,8]$, trauma $[9$, $10]$, brain tumors [11, 12], and hypertensive encephalopathy $[13,14]$.

Improvements in the imaging of water diffusion have been made by the development of the more complex diffusion tensor imaging (DTI), which allows direct examination, in vivo, of some aspects of tissue microstructure. DTI yields quantitative measures reflecting the integrity of white-matter fiber tracts, by taking advantage of the intrinsic directionality of water diffusion in human brain. The diffusion of water molecules is characterized by Brownian motion. When water molecules are unconstrained, the direction of motion of a given molecule is random. The displace- ments of water molecules over time are described by a Gaussian distribution. Diffusion is called isotropic when motion is equal and unconstrained in all directions. However, the microstructure of brain tissue forms physical boundaries that limit the Brownian motion of water molecules, resulting in restriction of the total amount of diffusion. In structures such as the whitematter fibers, the diffusion of water molecules will be relatively more restricted perpendicular to than parallel to the microstructural boundaries and diffusion is then called anisotropic [15].

In DWI, diffusion is described using a scalar parameter, the diffusion coefficient D. In tissues such as gray matter, where the measured apparent diffusivity is isotropic, it is sufficient to describe the diffusion characteristics with a single scalar apparent diffusion coefficient (ADC). In the presence of anisotropy, diffusion can no longer so be characterized, but requires a tensor $\mathrm{D}$, which fully describes the mobility of the molecules in each direction and the correlation between these directions. The mathematical construct used to characterize anisotropic Gaussian diffusion is a secondorder diffusion tensor [16]. Since the tensor is symmetric, 
at least six unique elements are required to fully characterize it [3]. The tensor can be diagonalized such that only three non-zero elements $\left(\lambda_{1}, \lambda_{2}\right.$, and $\left.\lambda_{3}\right)$ remain along the diagonal. These are known as the eigenvalues. Each eigenvalue is associated with an eigenvector $\left(\epsilon_{1}, \epsilon_{2}\right.$ and $\epsilon_{3}$ ), where the largest of the three eigenvalues $\left(\lambda_{1}\right)$ corresponds to the eigenvector $\epsilon_{1}$ and describes the principal direction of diffusion at that point.

The main artifacts in DTI data are the usual artifacts and problems associated with acquiring DWI data from which the diffusion tensor is estimated or measured. Artifacts include misregistration of data due to eddy currents, ghosting due to motion artifacts, and signal loss due to susceptibility variations. Hardware issues such as background gradients, gradient nonlinearity and miscalibration also have to be taken in consideration. However, improvements in image resolution and reduction of distortion have been made using motion-corrected multishot echoplanar imaging (EPI) PROPELLER and SENSE-EPI techniques [17, 18].

Diffusion tensor measurements result in a rich data set. Diffusion anisotropy can be measured in different ways by applying simple or more complicated mathematical formulae and recalculations using the underlying eigenvectors [3, 4, 16, 19]. A common way to summarize diffusion measurements in DTI is calculation of a parameters for overall diffusivity and for anisotropy. The ADC serves for overall diffusivity and is derived from the trace of the diffusion tensor, while anisotropy is represented by fractional anisotropy (FA) or relative anisotropy (RA). FA is a measure of the portion of the diffusion tensor due to anisotropy. The $\mathrm{RA}$ is derived from a ratio between the anisotropic and isotropic portions of the diffusion tensor. Another commonly used value is the volume ratio (VR) which expresses the relationship between the diffusion ellipsoid volume and that of a sphere, radius $\{\lambda\}$ [20]. Both water ADC and diffusion anisotropy differ markedly between childhood and adult brains, each varies with increasing age $[21,22,23]$.

The differences between these measurements lie in their sensitivity to anisotropy: FA is more sensitive to low and VR to high values of diffusion anisotropy, and RA scales linearly for different levels of anisotropy [3]. Both FA and RA are 0.0 for a purely isotropic medium. For higher symmetric anisotropic media FA tends towards 1 , while RA tends towards $\sqrt{2}$. Both FA and RA maps can be presented as gray-scale images. VR ranges from 1 (isotropic diffusion) to 0; some workers therefore prefer to use (1-VR). In contrast to the aforementioned measurements, which all represent intravoxel anisotropy, the lattice anisotropy index-another way of assessing diffusion-measures intervoxel anisotropy. The lattice measures of diffusion anisotropy allow neighboring voxels to be considered together in a region of interest, without losing anisotropy effects that result from different fiber orientations across voxels [20].

DTI allows us to look at anisotropic diffusion within white-matter tracts but is limited in demonstrating spatial, directional diffusion anisotropy. New and/or more sophisticated methods for demonstrating diffusion directions, such as color-coding and fiber tracking, have therefore been proposed [24, 25, 26, 27].

By choosing the eigenvector associated with the largest eigenvalue, the principal diffusion direction of the brain structure to be examined can be color-coded, resulting in color-coded maps or directionally encoded (DEC) FA maps. In these the fibers have been given different colors (blue for superior-inferior, green for anterior-posterior and red for left to right) depending on their direction of diffusion (Fig. 1). The magnitude of anisotropy, such as FA, can be used as an illumination factor of the calculation of a directionally coded color image [24].

In fiber tractography, or fiber tracking, white-matter tract directions are mapped on the assumption that in each voxel a measure of the local fiber orientation is obtained through diffusion tensor imaging. The task of tractography is sensibly to assign mathematical associations between adjacent voxels based on eigenvalue and eigenvector information. In DTI tractography it is assumed that the eigenvector associated with the largest eigenvalue is aligned with the direction of the fiber bundle [25, 26, 27, 28]. There are several problems associated with quantitative fiber tract tractography. First, all the usual artifacts and problems associated with DWI, as mentioned above, can adversely affect fiber tracking [27]. Interaction between vectors in the where fibers are crossing or "kissing" or where they branch or merge poses additional problems. A further problem is that there is no reference standard for in vivo tractography.

Since fiber tracking requires more extensive computer calculations and manpower than DWI or DTI it remains more of a research instrument and has, to date, little or no clinical application. However, it seems likely that tractography will improve our understanding of brain pathology, especially that of white-matter abnormalities. Possible applications include closed head injury and stroke, and even peripheral nerve injuries. Fiber tracking can be used in conjunction with functional MRI to analyze the anatomical connections and functional pathways for diagnostic purposes and presurgical planning [26].

\section{Clinical applications}

While DWI has several clinical applications and is routinely used in investigation of stroke, DTI is not routine in most institutions. However, an increased interest in 
Fig. 1a-d A healthy 30-yearold man. a Normal T2-weighted image $\mathbf{b}$ apparent diffusion coefficient (ADC) map c fractional anisotropy (FA) map d color-coded FA map. Highly directional white-matter structures are clearly seen on the FA maps. In $\mathbf{d}$ the fibers have been given different colors: blue for superior-inferior, green for anterior-posterior, and red for left to right, depending on their direction of diffusion
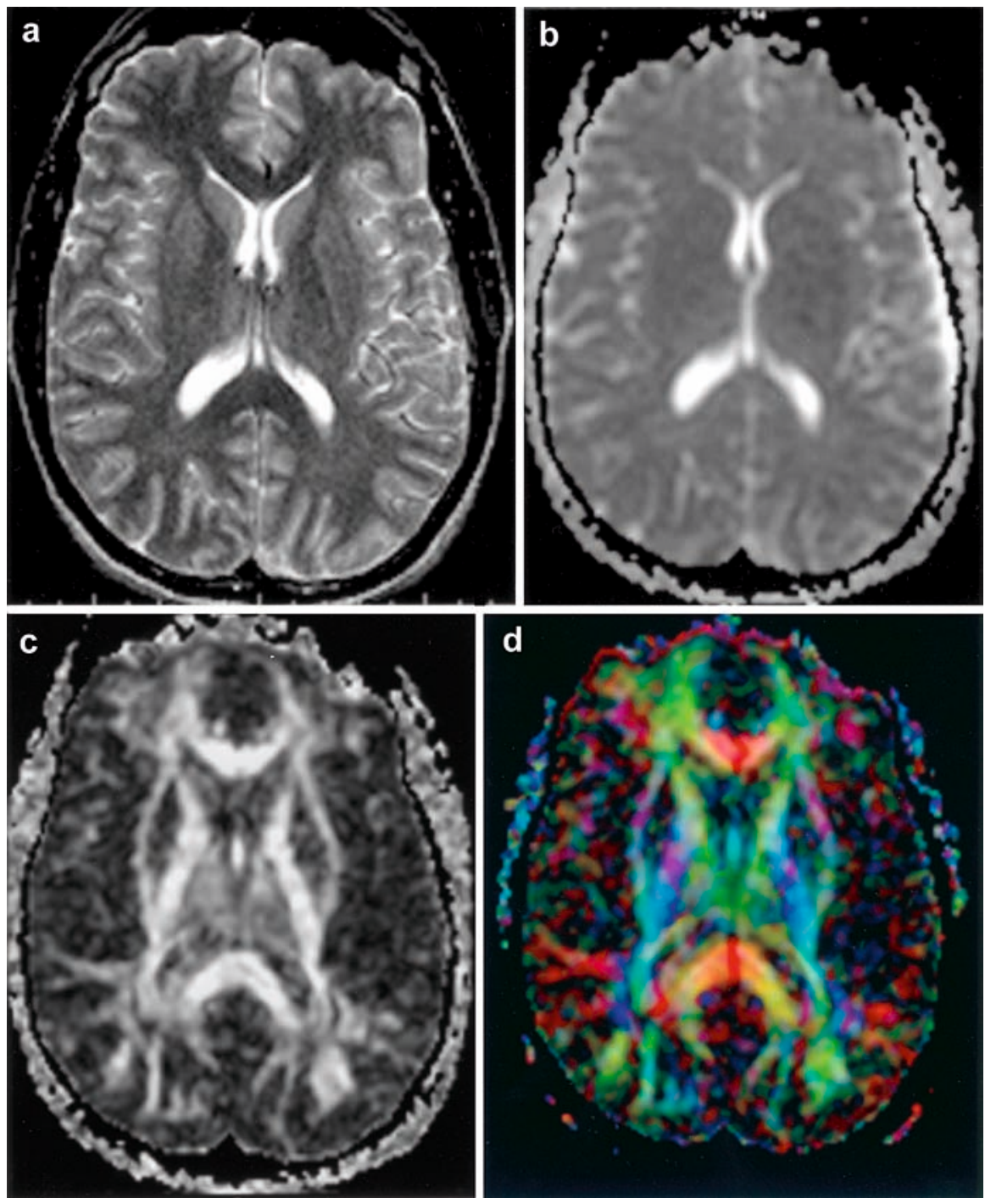

possible clinical applications and for evaluation of the value of DTI for examination of the brain has lead to intensive research and resulted in several reports. We reviews some clinical areas in which DTI has been shown to be of interest.

Cerebral ischemia, leukoariaosis and wallerian degeneration

A decrease in cerebral blood flow below $10-15 \mathrm{ml} / 100$ $\mathrm{g} / \mathrm{min}$ leads to an increased volume of intracellular water. This influx of water from the extracellular compartment causes the cells to swell producing cytotoxic edema. With conventional MRI, the acute stage of ischemia is poorly assessed and the extent of the ischemic parenchyma can be demonstrated only at a later stage, when vasogenic edema is present. DWI and DTI have been extensively used to detect acute ischemic brain injury while conventional MRI is still normal [1, 29, 30, 31]. They also make it possible to distinguish acute from chronic ischemic changes, which may have impact on treatment. In the acute phase, ADC are initially reduced by $30-50 \%$ within $30 \mathrm{~min}$ of the onset of focal ischemia $[29,30,31,32]$, more severely in the white than in the gray matter in acute and early subacute infarcts [33, 34]. With ischemic brain trauma, DTI parameters such as the $\mathrm{D}_{\mathrm{av}}$ (equivalent to $\mathrm{ADC}$ ), initially decrease but subsequently increase and become higher than normal. They remain elevated in the chronic phase of injury [35]. In the short interval between decreases and subsequent increases in ADC there is a time during which the values are normal, a process referred to as pseudonormalization. This occurs approximately 9 days after a cerebral infarct in adults and after 7 days in newborns [35]. In addition to the changes in the ADC, an acute elevation 
in FA has been observed in ischemic white but not grey matter. This acute elevation is followed by a marked reduction in FA during the chronic phase [36]. These changes are believed to be due to loss of organization in normal structures when their cytoarchitecture is disrupted.

In contrast to the renormalization and subsequent elevation of the ADC in chronic stroke, diffusion anisotropy remains significantly lower in the infarcted area than in the homologous contralateral region of the brain, even 2-6 months after an ischemic stroke [37]. ADC threshold values are useful in predicting tissue viability and stroke outcome [33, 38] and, combining ADC and anisotropy data, it is possible to assess the severity of strokes and predict outcome [33].

There are conditions under which ADC increase rather than decrease after an injury. In general, it appears that ADC decrease immediately after cell injury in the presence of cytotoxic edema. However it they be increased early when there is vasogenic edema, for example, in the reversible posterior leukoencephalopathy syndrome [13, 14], or in high-pressure hydrocephalus [39].

Leukoaraiosis is a nonspecific term for the radiological appearance of diffuse changes in the periventricular white matter seen on CT or MRI [40]. It may be seen in various diseases, including chronic ischemia, Alzheimer's disease, and CADASIL (cerebral autosomal dominant arteriopathy with subcortical infarcts and leukoencephalopathy): histologically, axonal loss and proliferation of glia has been reported [41, 42]. In ischemic leukoaraiosis, DTI shows elevated mean diffusivity and reduced FA in areas of increased signal on T2-weighted images [41]. Mean diffusivity in leukoaraiosis is significantly lower than that in a lacunar infarct, probably due to proliferation of glial tissue in the former, interfering with water diffusion [43].

Wallerian degeneration (WD) is as antegrade degeneration of axons and their myelin sheath secondary to proximal axonal injury or cell death, and most commonly involves the corticospinal tracts secondary to an ipsilateral cerebral infarct. DTI was more sensitive than T2-weighted imaging to WD [44]. Diffusion anisotropy was reduced both in the primary lesion and in the areas of $\mathrm{WD}$, whereas the ADC are only slightly increased in WD markedly increased in primary ischemic stroke lesions. ADC may thus have the potential to distinguish primary lesions from areas of WD [44].

\section{Developing brain, maturation and aging}

Several challenges exist for the application of DTI to the developing human brain. As mentioned above, the values of water ADC and diffusion anisotropy vary with age [21, 22, 23]. However, similar pulse sequences and post processing methods are used for both childhood and adult DTI with the exception of the $b$ value which is typically of the order of $1000 \mathrm{~mm}^{2} / \mathrm{s}$ for adults and $700-800 \mathrm{~mm}^{2} / \mathrm{s}$ for infants.

Significantly higher ADC and lower FA have been found in neonates than in adults [45]. The ADC have also been shown to be higher in the white than in the gray matter in childhood [21, 22]. ADC for the white matter of the centrum semiovale in premature infants approach $2.0 \times 10^{-3} \mathrm{~mm}^{2} / \mathrm{s}$. Thereafter, with increasing age, they decrease and the anisotropy values, especially the RA increase in a non-linear fashion during development until they reach the ADC of the adult brain, typically $0.7 \times 10^{-3} \mathrm{~mm}^{2} / \mathrm{s}[21,22]$ (Fig. 2). The changes in ADC occur predominantly occur within the first 6 months of life and are believed to be related to decreasing total water content, myelination, and the organization of the white-matter tracts, which all decrease diffusivity [46, 47, 48]. ADC change with increasing age and when measured in different parts of the brain $[45,49]$.

DTI has been used in the investigation of normal aging, to detect age-related degeneration [23, 50]. ADC are higher in the cerebral white matter of individuals older than 40 years of age than in younger individuals [51]. In addition, a decrease in diffusion anisotropy has been shown to occur after 20 years of age. Significant age-related declines in median FA have been demonstrated in densely packed white matter fiber areas, especially the genu of the corpus callosum and the centrum semiovale [23]. These interesting findings must be taken into account when assessing the effects of disease, especially in the elderly.

\section{Diffuse axonal injury}

Focal brain injuries other than stroke have not been widely studied with DTI, and it is not known whether mechanisms similar to those reported in ischemic stroke are involved in their recovery. Traumatic brain injuries can be classified as focal and diffuse. A focal head injury results from direct impact trauma, such as hematoma or cerebral contusion, whereas diffuse injuries result from shearing injuries and tensile strain on the brain as a result of rotation or deceleration of adjacent tissues that differ in density or rigidity. DWI can be used to show shearing injuries not visible on spin-echo or fluid attenuated inversion-recovery (FLAIR) T2-weighted images but is less sensitive than T2* imaging to hemorrhagic lesions [52]. In one study ADC maps were obtained in all patients and $65 \%$ of the DWI-positive lesions showed decreased diffusion [52] (Fig. 3). Surrounding a focal brain lesion such as a contusion or a focal hematoma, there is zone of "tissue at risk" defined by reduced diffusivity. This zone might be of importance in the development of new rescue therapy for patients 

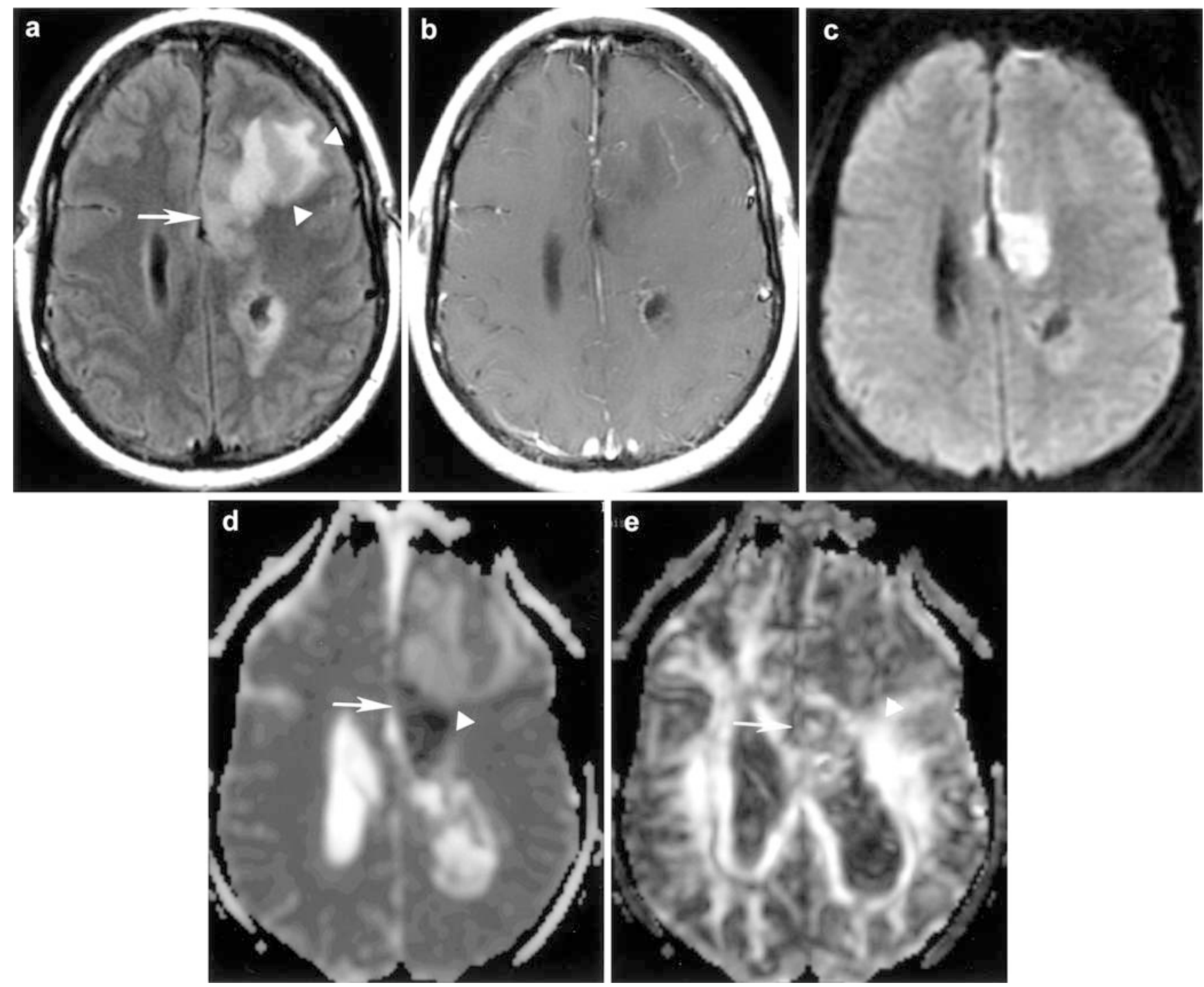

Fig. 2a-e A 45-year-old woman with a partly resected intraventricular ependymoma and a postoperative course complicated by left, dominant hemisphere ischemia, leading to aphasia. a A T2weighted fluid-attenuated inversion recovery (FLAIR) image demonstrates a slightly high-signal area medially in the left frontal lobe (arrow) and a large area of more increased signal in the deep subcortical and cortical regions of the left frontal lobe (arrowheads), which, on a contrast-enhanced T1-weighted image b gives low signal without obvious contrast enhancement. c Diffusionweighted imaging (DWI) shows an area of restricted diffusion consistent with acute ischemia. The ADC were decreased $\left(0.50 \pm 06 \times 10^{-9} \pm \mathrm{mm}^{2} / \mathrm{s}\right)$ in this area (arrow) and $\mathbf{d}$ increased $\left(1.5 \pm 0.05 \times 10^{-9} \mathrm{~mm}^{2} / \mathrm{s}\right)$ in an area of chronic ischemia (arrowhead). FA was decreased $(0.22 \pm 0.05)$ in the area of acute ischemia (arrow) and even more so $(0.07 \pm 0.02)$ in the area of chronic ischemia (arrowhead)

with head trauma [53]. Most of the histopathologic abnormalities seen in with altered diffusion anisotropy appear to be in the internal capsule and corpus callosum. The significant reduction in diffusion anisotropy seen in

white matter appearing normal on conventional MRI in the first $24 \mathrm{~h}$ after diffuse axonal injury will be less evident, although still abnormal weeks after the trauma [54]. A decrease in diffusion coefficient have been demonstrated in normal or almost normal appearing areas on T1-weighted and spin-echo or FLAIR T2-weighted images in the first day of life in newborns with high risk of perinatal brain injury. However, this decrease became more obvious on the third day of life, with pseudonormalization of the values within a week, by which time conventional MRI was abnormal. This suggests that DTI on the first day after trauma might not show the full extent of the injury in a neonate and that images 3 days after the injury might be better predictors of the final extent of the damage [55]. Further research in this area is needed to fully evaluate the use of DTI to estimate the full extent of a brain injury, optimal timing for MRI and prediction of outcome. 
Fig. 3a-d A 23-year-old man with a severe brain injury, unresponsive after a motor vehicle accident. a T2-weighted FLAIR and b T2-weighted images $30 \mathrm{~h}$ after the accident demonstrate high signal in the body and splenium of the corpus callosum, without hemorrhage, consistent with axonal shearing. c, d ADC were increased $\left(1.1 \pm 0.06 \times 10^{-9} \mathrm{~mm}^{2} / \mathrm{s}\right)$ (arrow) and FA was decreased $(0.38 \pm 0.05)($ arrow $)$
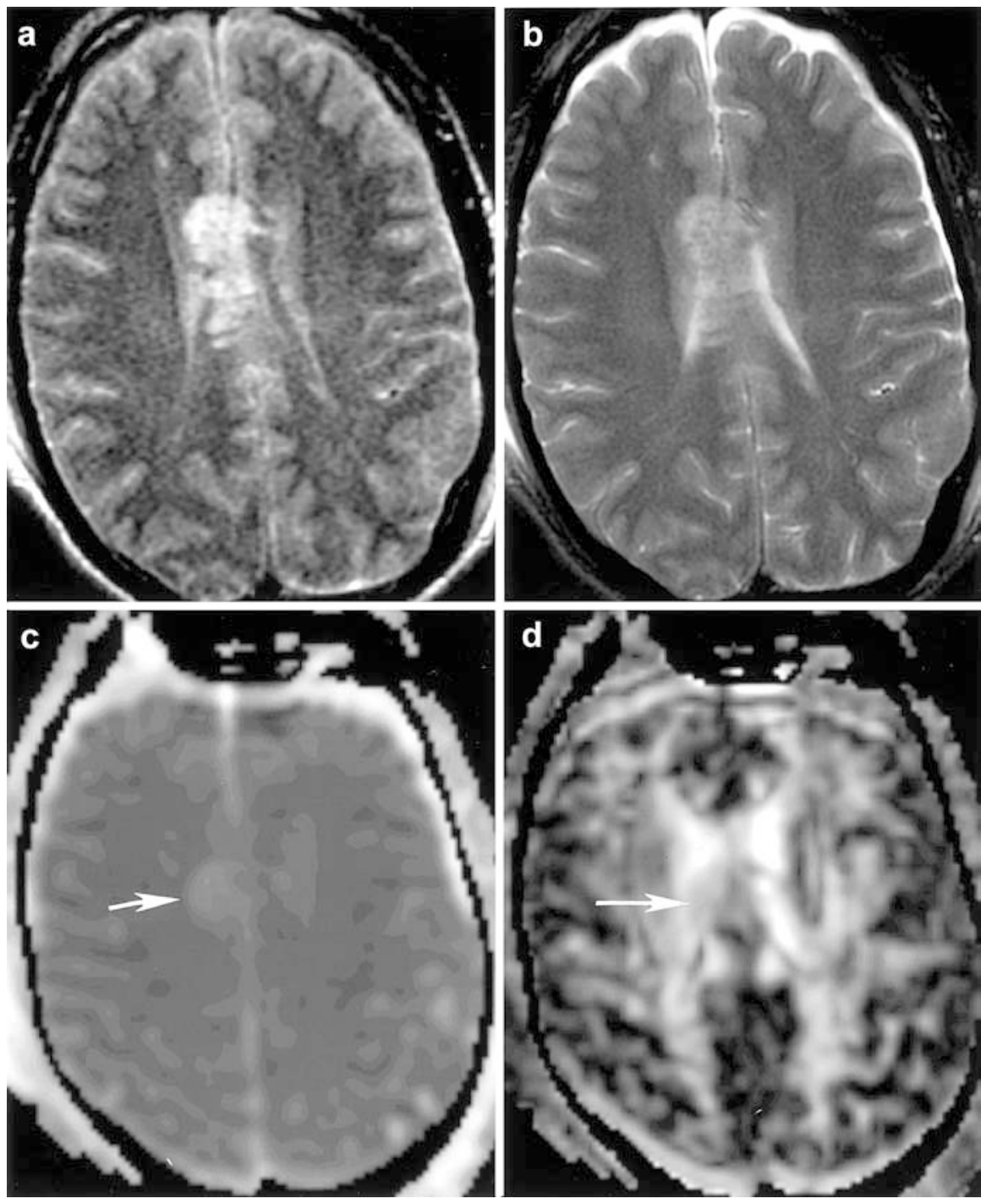

\section{Multiple sclerosis}

Numerous studies on multiple sclerosis (MS) have shown that mean diffusivity is elevated in the lesions seen on T2- weighted images. The degree of elevation seems to be related to the clinical course of the disease. The lesions with more "destructive" pathology are generally shown to have the highest diffusivity $[7,50,56$, $57,58]$. Increased diffusivity $(4-13 \%)$ has also been found in normal-appearing white matter of patients with MS, which may suggest that it is a diffuse white matter disease as well as multifocal. On the other hand, this may also suggest that the white matter is subject to wallerian degeneration proximal and distal to the visible lesions [7, 8, 57, 58, 59]. A recent study has demonstrated increased water diffusivity in the cerebral gray matter of patients with MS, suggesting that the gray matter may not be spared by the pathological process [60].
It would appear that the FA is more sensitive than ADC values to white-matter abnormalities in MS [57, 61, 62]. Some studies have demonstrated generally reduced diffusion anisotropy in MS plaques (Fig. 4); in one, the lowest anisotropy was seen in acute lesions [57] while in another, the lowest anisotropy was found in "black holes", the lesions giving low signal on T1-weighted images [58].

\section{Epilepsy}

A common cause of refractory complex partial seizures arising in the temporal lobes is hippocampal sclerosis [63]. High-resolution MRI has been used to identify specific features such as hippocampal volume loss and high signal on spin-echo or FLAIR T2-weighted images $[64,65]$. Recent studies applying DTI to patients with chronic epilepsy and hippocampal sclerosis have 

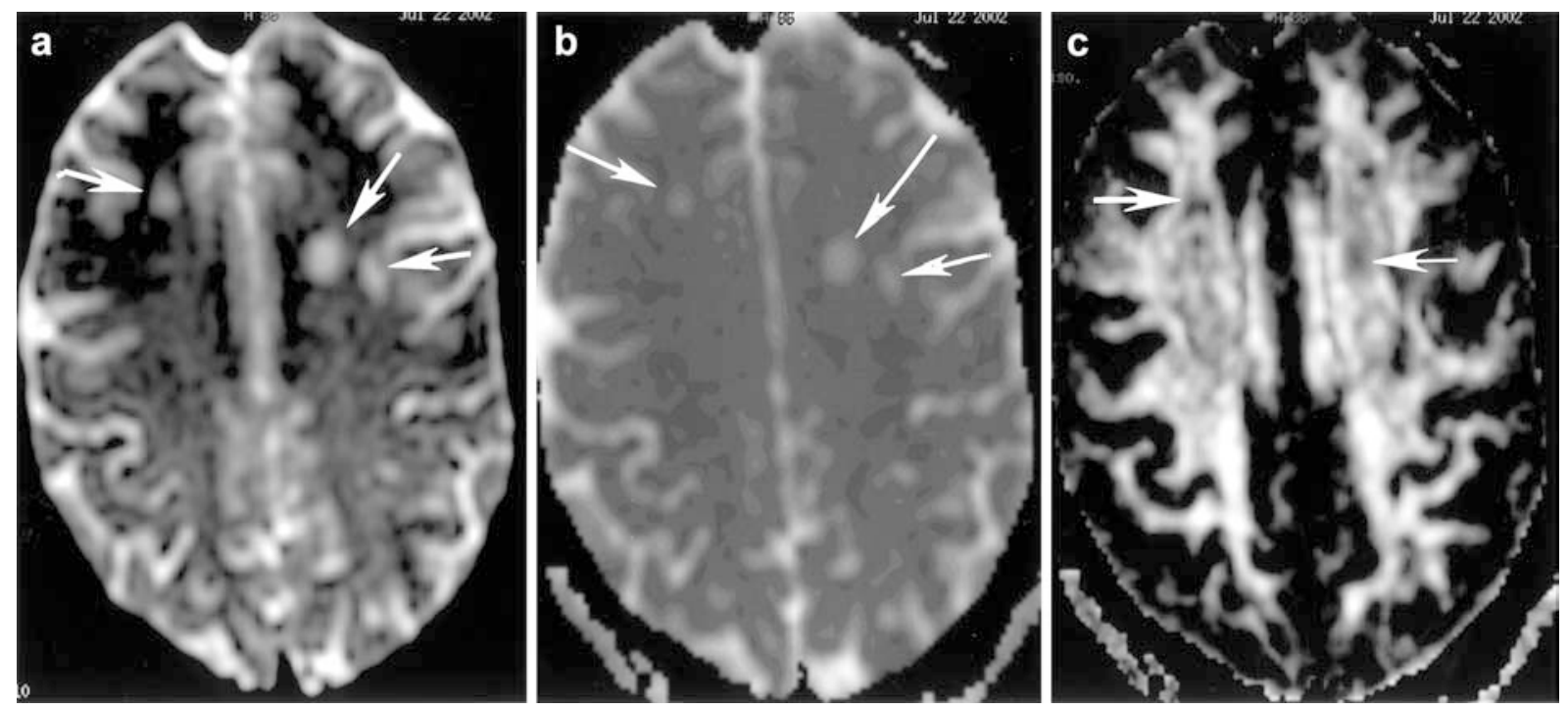

Fig. 4a-c A 45-year-old woman with multiple sclerosis. a A T2-weighted image demonstrates areas of increased signal representing plaques (arrows). These show b increased ADC (arrows) and $\mathbf{c}$ decreased FA (arrows)

demonstrated increased diffusivity and reduced anisotropy in sclerotic hippocampi, suggesting loss of structural organization and expansion of the extracellular space $[66,67]$. The changes in diffusion extend to involve normal-appearing brain [67]. In contrast to these patients, high-resolution techniques have been used to identify malformations of cortical development (MCD) as potential epileptogenic foci in patients with refractory extratemporal, neocortical epilepsy. Preliminary studies of in 12 patients with refractory epilepsy and cortical signal abnormalities showed differences in ADC within the lesion and contralateral normal brain in the same location; there was a significant overlap between the FA [68]. Several investigations using DTI revealed higher diffusivity and lower anisotropy in the zones of MCD [69], and in surrounding brain that appeared normal on conventional MRI $[69,70]$. DTI may prove valuable for identifying epileptogenic foci as well as more optimally defining the extent of the lesion for surgical resection.

\section{Alzheimer's disease (AD)}

Studies of patients with a clinical diagnosis of AD have concentrated on changes in anisotropy associated with progression of the disease. Changes in diffusion anisotropy have been demonstrated by measuring diffusivity in the corpus callosum in the midline, where axons are predominantly oriented transversely. One study showed that anisotropy was lower in the genu and splenium in patients with presumed AD than in sex- and age-matched controls, probably due to axonal loss or demyelination in these areas [71]. In another study the integrity of axonal tracts in areas associated with cognitive function was compared with that of tracts associated with motor function [72]. Using the lattice index [20] as a measure of white-matter tract integrity, the motor tracts appeared preserved, but there was presumed axonal degeneration in the cognitive tracts [72].

DTI has also been used in the investigation of other forms of cognitive impairment, such as in adults with reading difficulties [73], and for detecting degenerate fiber tracts in the disconnection syndrome [74]. A recent investigation showed decreased diffusion anisotropy bilaterally in the temporoparietal white matter in patients with reading difficulty. White-matter diffusion anisotropy in the left temporoparietal region correlated significantly with the reading scores of the reading-impaired adults and a control group [73], presumably reflecting the microstructure of white-matter tracts, which may contribute to reading ability.

\section{Brain tumors}

Radiological specification and grading of a brain tumor is limited, although conventional MRI can be used to demonstrate the site and extent of the tumor. There has been an increasing interest in the use of DWI and DTI to identify different tumor components, and to differentiate tumor invasion from normal brain tissue or edema. ADC maps have proved helpful in defining solid, enhancing tumor, nonenhancing lesion, peritumoral edema, and necrotic and/or cystic regions from normal surrounding brain.

Cystic or necrotic regions have the highest ADC [75] while the contrast-enhancing part of a tumor has lower ADC than the cystic or necrotic areas and the edema $[11,12,75,76,77,78,79,80]$. Significantly higher mean diffusivity and lower FA than in normal-appearing white 
Fig. 5a-d A 53-year old man who had received radiation therapy for a left posterior frontal glioblastoma multiforme. a T2-weighted (arrows) and $\mathbf{b}$ contrast-enhanced T1-weighted images show an enhancing lesion adjacent to the left lateral ventricle. In region of interest 2 ADC are slightly increased $\left(0.87 \pm 0.08 \times 10^{-}\right.$ ${ }^{9} \mathrm{~mm}^{2} / \mathrm{s}$ ), compared to the normal region of interest 3 $\left(0.76 \pm 0.03 \times 10^{-9} \mathrm{~mm}^{2} / \mathrm{s}\right.$ (arrows). d FA are decreased $(0.09 \pm 0.02)$ compared to $(0.5 \pm 0.04)$ (arrows)
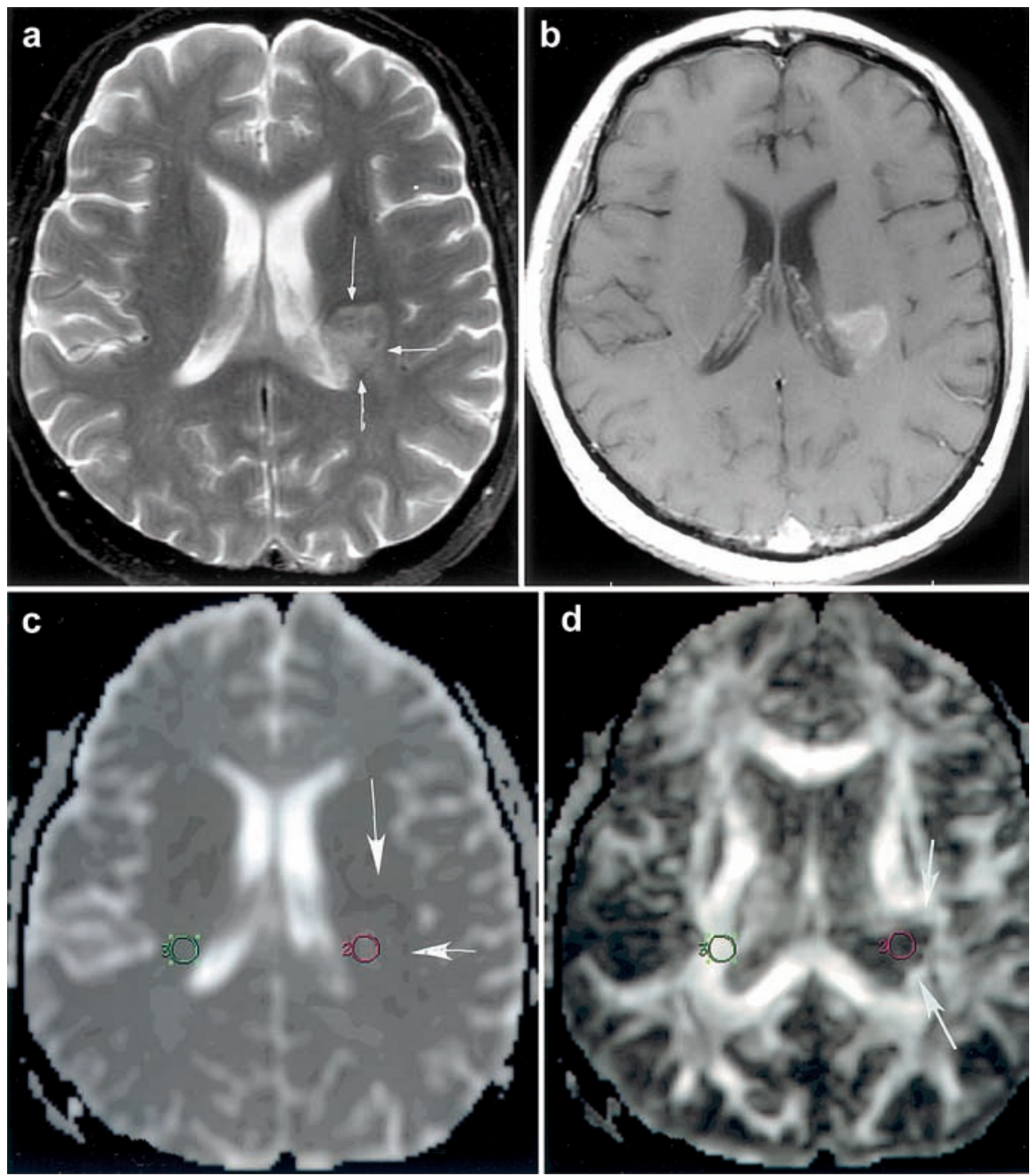

matter have been demonstrated in the peritumoral region of both gliomas and metastases. Peritumoral mean diffusivity of metastases was significantly higher than that of gliomas whereas the FA was similar, suggesting that the FA changes surrounding gliomas can be attributed to both increased water content and tumor infiltration [81].

The possibility of determining the type and grade of a tumor using DWI and DTI has been explored in both adults and children. Low-grade astrocytomas have higher and high-grade malignant gliomas lower ADC values, reflecting more restricted diffusion with increasing tumor cellularity $[12,77,78,82]$. However a recent study showed that FA values, which are generally reduced in tumors, suggesting structural disorder, do not add additional information for tissue differentiation [79], but may help in the understanding of the effect of brain tumors on white-matter fibers, which might be important in surgical planning [83, 84] (Fig. 5).

Obtaining reliable information about tumor response to therapy is crucial to treatment. New data from both animal models and human studies suggest that diffusion imaging may be sensitive to tumor response to therapy [85, 86, 87]. An early increase in ADC during therapy may relate to therapy-induced cell necrosis. A subsequent fall in ADC to pretreatment levels within the tumor is thought to indicate tumor regrowth.

\section{Metabolic disorders}

Reports on DTI in metabolic disorders, such as adrenoleukodystrophy (ALD) [88, 89, 90], and Krabbe's disease [91], and in the differentiation of dysmyelinating from the demyelinating disorders [92, 93] have been published. In X-linked ALD a decrease in FA and an increase in ADC have been demonstrated [89, 90], correlating with the well-recognised histopathological zones described in this disease [94] (Fig. 6). These findings suggest increased diffusivity due to an increase in free water and loss of the integrity of the myelin sheaths and axons in the white matter. 

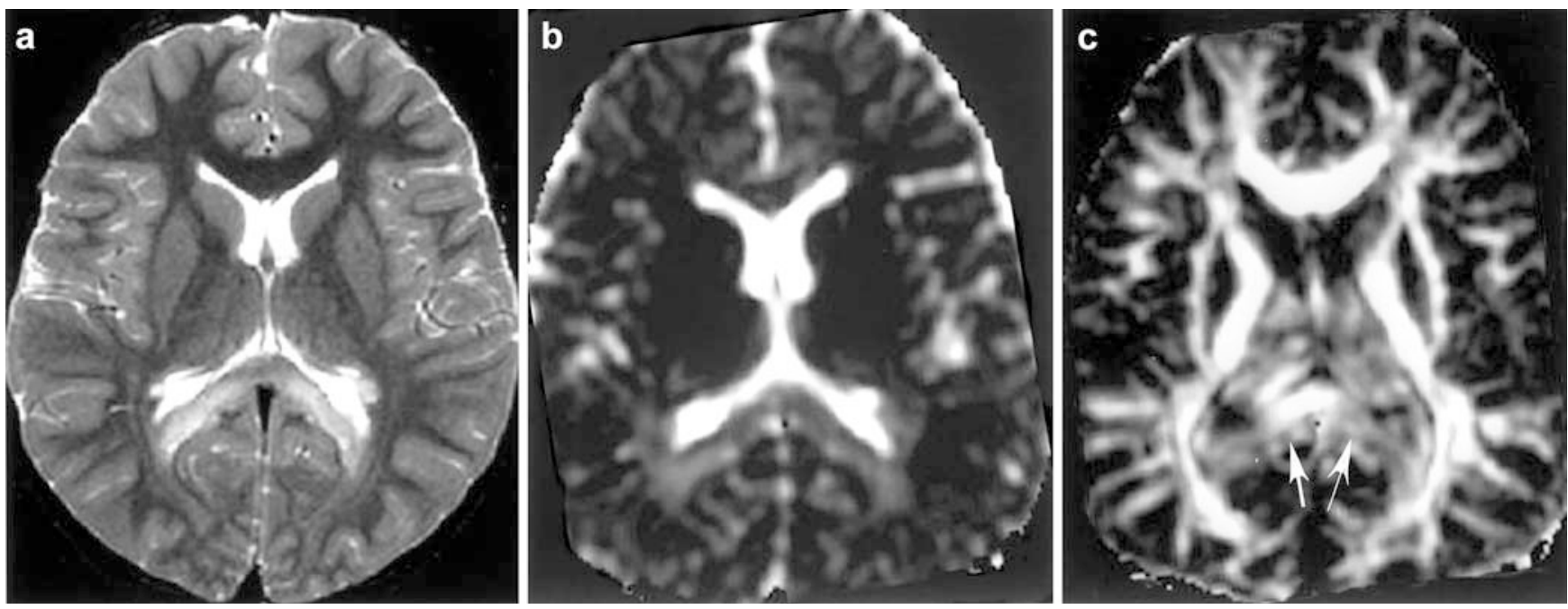

Fig. 6a-c A 6-year-old boy with x-linked adrenoleukodystrophy. a High signal on a T2-weighted image extends along the splenium the of corpus callosum bilaterally, with minimal extension into the peritrigonal white matter of both occipital lobes. b ADC are markedly increased ADC, while c FA is decreased. On the FA map the splenium of the corpus callosum appears darker (arrows) than the genu of the corpus callosum

DTI has been found to be superior to conventional MRI in differentiating dysmyelinating disorders, such as Pelizaeus-Merzbacher disease from demyelinating disorders, such as Krabbe's disease and Alexander's disease. Diffusional anisotropy is present in dysmyelinated lesions but is lost in demyelinated lesions; these findings have been verified in both humans and in mice with conditions thought to parallel the human diseases [92, 93].

Recent reports describe the use of DTI to characterize and diagnose other diseases such as amyotrophic lateral sclerosis [95], hereditary disorders such as CA-

\section{Conclusions}

The recent development of DTI allows direct examination, in vivo, of some aspects of brain microstructure. DTI has already shown to be of value in studies of neuroanatomy, fiber connectivity, and brain development. It has become interesting for investigation of different brain pathology, such as cerebral ischemia, trauma, MS, presumed AD and cognitive impairment, epilepsy, brain tumors and metabolic disorders. However, further improvement in technique and stable postprocessing analyses is needed to increase the utility of DTI in both research and clinical applications.

Acknowledgements We wish to say a special thank you to Professor Thomas Chenevert, Department of Radiology, University of Michigan Hospitals, for valuable comments and support in the preparation of this manuscript.

\section{References}

1. Moseley M, Cohen Y, Kucharczyk J, et al (1990) Diffusion-weighted MR-imaging of anisotropic water diffusion in cat central nervous system. Radiology 176: 439-445

2. Le Bihan D (1991) Molecular diffusion nuclear magnetic resonance imaging. Magn Reson Q 7: 1-30

3. Bammer R (2003) Basic principles of diffusion-weighted imaging. Eur J Radiol 45: 169-184
4. Moseley ME, Kucharczyk J, Mintorovitch J, et al (1990) Diffusionweighted MR imaging of acute stroke: correlation with T2-weighted and magnetic susceptibility-enhanced MR imaging in cats. AJNR 11: 423-429

5. Lansberg MG, Norbash AM, Marks MP, Tong DC, Moseley ME, Albers GW (2000) Advantages of adding diffusion-weighted magnetic resonance imaging to conventional magnetic resonance imaging for evaluating acute stroke. Arch Neurol 57: 1311-1316
6. Larsson HBW, Thomsen C, Fredriksen J, Stubgaard M, Henriksen O (1992) In vivo magnetic resonance diffusion measurements in the brain of patients with multiple sclerosis. Magn Reson Imaging 10: 7-12

7. Horsfield MA, Lai M, Webb S, et al (1996) Apparent diffusion coefficients in benign and secondary progressive multiple sclerosis by nuclear magnetic resonance. Magn Reson Med 36: 393-400 
8. Christensen P, Gideon P, Thomsen C, Stubgaard M, Henriksen O, Larsson HBW (1993) Increased water self-diffusion in chronic plaques and in apparently normal white matter in patients with multiple sclerosis. Acta Neurol Scand 87: 195-197

9. Nakahara M, Ericsson K, Bellander BM (2001) Diffusion-weighted MR and apparent diffusion coefficient in the evaluation of severe brain injury. Acta Radiol 42: 365-369

10. Sundgren PC, Reinstrup P, Romner B, Holtås S, Maly P (2002) Value of conventional, and diffusion-and perfusion weighted MRI in the management of patients with unclear cerebral pathology, admitted to the intensive care unit. Neuroradiology 44 : 674-680

11. Stadnik T, Chaskis C, Michotte A, et al (2001) Diffusion-weighted MR imaging of intracerebral masses: comparison with conventional MR imaging and histologic findings. ANJR 22: 969-976

12. Kono K, Inoue $\mathrm{Y}$, Nakayama $\mathrm{K}$, et al (2001) The role of diffusion-weighted imaging in patients with brain tumors. AJNR 22: 1081-1088

13. Sundgren $\mathrm{PC}$, Edvardssson $\mathrm{B}$, Holtås $\mathrm{S}$ (2002) Serial investigation of perfusion disturbances and vasogenic oedema in hypertensive encephalopathy by diffusion and perfusion weighted imaging. Neuroradiology 44 299-304

14. Hinchey J, Chaves C, Applgnani B, (1996) A reversible posterior leukoencephalopathy syndrome. N Engl J Med 334: 494-500

15. Le Bihan D (ed.) (1995) Diffusion and perfusion magnetic resonance imaging. Raven Press, New York

16. Le Bihan, D, Mangin JF, Poupon C, Clark CA, Pappata S, Molko N (2001) Diffusion tensor imaging: concepts and applications. Magn Reson Imaging 13: 534-546

17. Pipe JG, Farthing VG, Forbes KP (2002) Multishot diffusion FSE using PROPELLER MRI. Magn Reson Med 47: 42-52

18. Bammer R, Auer M, Keeling SL, et al (2002) Diffusion tensor imaging using single shot SENSE-EPI. Magn Reson Med 48: 123-136

19. Basser PJ, Jones DK (2002) Diffusion tensor MRI: theory, experimental design and data analysis - a technical review. NMR Biomed 14: 456-467

20. Pierpaoli C, Basser PJ (1996) Toward a quantitative measurement of diffusion anisotropy. Magn Reson Med 36: 893-906
21. Mukherjee P, Miller JH, Shimony JS et al (2001) Normal brain maturation during childhood: developmental trends characterized with diffusion-tensor MR imaging. Radiology 221: 349-358

22. Morris MC, Zimmerman RA, Bilaniuk LT, Hunter JV, Hasselgrove JC (1999) Changes in brain water diffusion during childhood. Neuroradiology 41: 929-934

23. Pfefferbaum, A, Sullivan EV, Hedehus M, Lim KO, Adalsteinsson E, Moseley M (2000) Age-related decline in brain white matter anisotropy measured with spatially corrected echo-planar diffusion tensor imaging. Magn Reson Med 44: 259-268

24. Pajevic S, Pierpaoli C (1999) Color schemes to represent the orientation of anisotropic tissues from diffusion tensor data; application to white matter fiber tract mapping in the human brain. Magn Reson Med 42: 526-540

25. Mori S, Van Zijl PC (2002) Fiber tracking: principles and strategies - a technical review. NMR Biomed 15: 468-480

26. Lori NF, Akbudak E, Shimony JS, et al (2002) Diffusion tensor fiber tracking of human brain connectivity: acquisition methods, reliability analysis and biological results. NMR Biomed 15: 493 515

27. Basser PJ, Pajevic S, Pierpaoli C, Duda J, Aldroubi A (2000) In vivo fiber tractography using DT-MRI data. Magn Reson Med 44: 625-632

28. Bammer R, Acar, B, Moseley M (2002) In vivo MR tractography using diffusion imaging. Eur J Radiol 45: 223-234

29. Sorensen AG, Buonanno FS, Gonzalez RG, (1996) Hyperacute stroke: evaluation with combined multisection diffusion-weighted and hemodynamically weighted echo-planar MR imaging. Radiology 199: 391-401

30. Lutsep HL, Albers GW, DeCrespigny A, Kamat GN, Marks MP, Moseley ME (1997) Clinical utility of diffusionweighted magnetic resonance imaging in the assessment of ischemic stroke. Ann Neurol 41: 574-580

31. Zelaya F, Flood N, Chalk JB, et al (1999) An evaluation of the time dependence of the anisotropy of the water diffusion tensor in acute human ischemia. Magn Reson Imaging 17: 331-348

32. Weber J, Mattle HP, Heid O, Remonda L, Schroth G (2000) Diffusion-weighted imaging in ischaemic stroke: a follow-up study. Neuroradiology 42: 184-191

33. Yang Q, Tress BM, Barber PA, et al (1999) Serial study of apparent diffusion coefficient and anisotropy in patients with acute stroke. Stroke 30: 2382-2390
34. Mukherjee P, Bahn MM, McKinstry RC, et al (2000) Differences between gray matter and white matter diffusion in stroke: diffusion-tensor MR imaging in 12 patients. Radiology 215: 211-220

35. Copen WA, Schwamm LH, Gonzalez $\mathrm{RG}$, et al (2001) Ischemic stroke: effects of etiology and patient age in the time course of the core apparent diffusion coefficient. Radiology 221: 27-34

36. Sorensen AG, Wu O, Copen WA, et al (1999) Human acute cerebral ischemia: detection of changes in water diffusion anisotropy by using MR imaging. Radiology 212: 785-792

37. Werring DJ, Toosy AT, Clark CA, et a (2000) Diffusion tensor imaging can detect and quantify corticospinal tract degeneration after stroke. J.Neurol Neurosurg Psychiatry 69: 269-272

38. Schlaug G, Siewert B, Benfield A, Edelman RR, Warach S (1997) Time course of the apparent diffusion coefficient (ADC) abnormality in human stroke. Neurology 49: 113-119

39. Gideon P, Stahlberg F, Thomsen C, Gjerris F, Sorensen PS, Henriksen O (1994) Cerebrospinal fluid flow and production in patients with normal pressure hydrocephalus studied by MRI. Neuroradiology 36: 210-215

40. Hachinski VC, Potter P, Merskey H (1987) Leuko-araiosis. Arch Neurol 44: 21-33

41. Brown MM (1995) Leukoaraiosis. In: Dooan GA, Norrving B, Bamford JM, Bogousslavsky J (eds) Lacunar and other subcortical infarctions Oxford University Press, Oxford, pp 47-66

42. Pantoni L, Garcia JH (1997) Pathogenesis of leukoaraiosis: a review. Stroke 28: 652-659

43. Jones DK, Lythgoe D, Horsfield MA, Simmons A, Williams SCR, Markus HS (1999) Characterization of white matter damage in ischemic leukoaraiosis with diffusion tensor MRI. Stroke 30: 393-397

44. Pierpaoli C, Barnett A, Pajevic S, et al (2001) Water diffusion changes in Wallerian degeneration and their dependence on white matter architecture. NeuroImage 13: 1174-1185

45. Nell J, Shiran S, McKinstry R, et al (1998) Normal brain in human newborns: Apparent diffusion coefficient and diffusion anisotropy measured by using diffusion tensor MR imaging. Radiology 209: 57-66

46. Wimberger D.M, Roberts T.R, Barkovitch A.J, Prayer LM, Moseley ME, Kucharczyk J (1995) Identification of "premyelination" by diffusion-weighted MRI. J Comput Assist Tompogr 19: 28-33 
47. Sakuma H, Nomura Y, Takeda K, et al (1991) Adult and neonatal human brain: diffusional anisotropy and myelination with diffusion-weighted MR imaging. Radiology 180: 229-233

48. Nomura Y, Sakuma H, Takeda K, Tagami T, Okuda Y, Nakagawa T (1994) Diffusion anisotropy of the human brain assessed with diffusion-weighted MR: relation with normal brain development and aging. AJNR 15: 231-238

49. Huppi P, Maier S, Peled S, et al (1998) Microstructural development of human newborn cerebral white matter assessed in vivo by diffusion tensor MRI. Pediatr Res 44:584-5901

50. Nusbaum AO, Lu D, Tang CY, Altas SW (2000) Quantitative diffusion measurements in focal multiple sclerosis lesions: correlations with appearance on T1-weighted images. Am J Roentgenol 175: $821-825$

51. Gideon P, Thomsen C, Henriksen O (1994) Increased self-diffusion of brain water in normal aging. Magn Reson Imaging 4: 185-188

52. Huisman AGM, Sorensen AG, Hergan K, Gonzalez RG, Schaefer PW (2003) Diffusion-weighted imaging for the evaluation of diffuse axonal injury in closed head injury. J Comput Assist Tomogr 27: 5-11

53. Jones DK, Dardis R, Ervine M, et al (2000) Cluster analysis of diffusion tensor magnetic resonance images in human head injury. Neurosurgery 47: $306-$ 317

54. Arfanakis K, Haughton VM, Carew JD, Rogers BP, Dempsey RJ, Meyerand ME (2002) Diffusion tensor MR imaging in diffuse axonal injury. AJNR 23: 794-802

55. Mckinstry RC, Miller JH, Snyder AZ, et al (2003) A prospective, longitudinal diffusion tensor imaging study of brain injury in newborns. Neurology 59: 824-833

56. Droogan AG, Clark CA, Werring DJ, Barker GJ, McDonald WI, Miller DH (1999) Comparison of MS clinical subgroups using navigated diffusionweighted imaging. Magn Reson Imaging 17: 653-661

57. Werring DJ, Clark CA, Barker GJ, Thompson AJ, Miller DH (1999) Diffusion tensor imaging of lesions and normal-appearing white matter in multiple sclerosis. Neurology 52: 1626-1632

58. Bammer R, Augustin M, Strasser-Fuchs $S$, et al (2000) Magnetic resonance diffusion tensor imaging for characterizing diffuse and focal white matter abnormalities in multiple sclerosis. Magn Reson Med 44: 583-591
59. Filippi M, Ianucci G, Cercignani M, Rocca MA, Praseti A, Comi G (2000) A quantitative study of water diffusion in multiple sclerosis lesions and normalappearing white matter using echo-planar imaging. Arch Neurol 57: 10171021

60. Bozzali M, Cercignani M, Sormani M, Comi G, Filippi M (2002) Quantification of brain gray matter damage in different MS phenotypes by use of diffusion tensor MR imaging. AJNR 23: 985-988

61. Guo A, MacFall J, Provenzale J (2002) Multiple sclerosis; diffusion tensor MR imaging for evaluation of normalappearing white matter. Radiology 222: 729-736

62. Filippi M, Cercignani M, Inglese $M$, Horsfield M, Comi G (2001) Diffusion tensor magnetic resonance imaging in multiple sclerosis. Neurology 56: 304 311

63. Brown WJ, Babb TL (1987) Neuropathological changes in the temporal lobe associated with complex partial seizures. In Hopkins A (ed) Epilepsy. Demos, New York, pp 300-323

64. Brooks BS, King DW, el Gammal T, et al (1990) MR imaging in patients with intractable complex partial epileptic seizures. ANJR 11: 93-99

65. Tien R, Felsberg G, Castro C, et al (1993) Complex partial seizure and mesial temporal sclerosis: evaluation with fast spin-echo MR imaging. Radiology 189: 835-842

66. Wieshman UC, Clark CA, Symms MR, Franconi F, Barker GJ, Shorvon SD (1999) Reduced anisotropy of water diffusion in human hippocampus in epilepsy. Magn Reson Imaging 17: 29-36

67. Yoo SY, Chang KH, Song IC, et al (2002) Apparent diffusion coefficient value of the hippocampus in patients with hippocampal sclerosis and in healthy volunteers. ANJR 23: 809-812

68. Eriksson SH, Rugg-Gunn FJ, Symms MR, Barker GJ, Duncan JS (2001) Diffusion tensor imaging in patients with epilepsy and malformations of cortical development. Brain 124: 617626

69. Arfanakis K, Hermann BP, Rogers BP, Carew JD, Seidenberg M, Meyerand ME (2002) Diffusion tensor MRI in temporal lobe epilepsy. Magn Reson Imaging 20: 511-519

70. Rugg-Gunn FJ, Eriksson SH, Symms MR, Barker GJ, Duncan JS (2001) Diffusion tensor imaging of cryptogenic and acquired partial epilepsies. Brain 124: 627-636
71. Hanyu H, Sakurai H, Iwamoto T, Takasaki M, Shindo H, Abe K (1998) Diffusion-weighted MR imaging of the hippocampus and temporal white matter in Alzheimer's disease. J Neurol Sci 156: 195-200

72. Rose SE, Chen F, Chalk JB, et al (2000) Loss of connectivity in Alzheimer's disease: an evaluation of the white matter tract integrity with color coded MR diffusion tensor imaging. J Neurol Neurosurg Psychiatry 69: 530

73. Klingberg T, Hedehus M, Temple E, et al (2000) Microstructure of temporo-parietal white matter as a basis for reading ability: evidence from diffusion tensor magnetic resonance imaging. Neuron 25: 493-500

74. Molko N, Cohen L, Mangin JF, et al (2002) Visualizing the neural bases of a disconnection syndrome with diffusion tensor imaging. J Cogn Neurosci 14: 629-636

75. Brunberg J, Chenevert T, McKeever PE, et al (1995) In vivo MR determination of water diffusion coefficients and diffusion anisotropy: correlation with structural alteration in gliomas of the cerebral hemispheres. ANJR 16: 361-371

76. 76 Krabbe K, Gideon $P$, Wagn $P$, Hansen U, Thomsen C, Madsen F (1997) MR diffusion imaging of human intracranial tumours. Neuroradiology 1997;39:483-489

77. Castillo M, Smith J, Kwock L, Wilbert K (2001) Apparent diffusion coefficients in the evaluation of high-grade cerebral gliomas. ANJR 22: 60-64

78. Guo A, Cummings T, Dash R, Provenzale J (2002) Lymphomas and high-grade astrocytomas: comparison of water diffusibility and histologic characteristics. Radiology 224: 177-183

79. Sinha S, Bastin ME, Whittle IR, Wardlaw JM (2002) Diffusion tensor imaging of high-grade cerebral gliomas. AJNR 23: 520-527

80. Bastin M, Sinha S, Whittle I, Wardlaw J (2002) Measurements of water diffusion and $\mathrm{T} 1$ values in peritumoural oedematous brain. Neuroreport 13:13351340

81. Lu S, Ahn D, Johnson G, Cha S (2003) Peritumoral diffusion tensor imaging of high-grade gliomas and metastatic brain tumors. AJNR 24: 937-941

82. Gauvain K, McKinstry R, Mukherjee $\mathrm{P}$, et al (2001) Evaluating pediatric brain tumor cellularity with diffusion tensor imaging. Am J Roentgenol 177: 449-454 
83. Wieshmann UC, Symms MR, Parker GJ, et al (2000) Diffusion tensor imaging demonstrates deviation of fibres in normal appearing white matter adjacent to a brain tumour. J Neurol Neurosurg Psychiatry 68: 501-503

84. Mori S, Fredriksen K, van Zijl PC, et al (2002) Brain white matter anatomy of tumor patients evaluated with diffusion tensor imaging. Ann Neurol 51: 377-380

85. Chenevert TL, McKeever PE, Ross BD (1997) Monitoring early response of experimental brain tumors to therapy using diffusion magnetic resonance imaging. Clin Cancer Res 3: 1457-1466

86. Chenevert TL, Stegman LD, Taylor JM (2000) Diffusion magnetic resonance imaging; an early surrogate marker of therapeutic efficacy in brain tumors. J Natl Cancer Inst 92: 2029-2036

87. Mardor Y, Pfeffer R, Spiegelmann R, et al (2003) Early detection of response to radiation therapy in patients with brain malignancies using conventional and high $b$-value diffusion weighted magnetic resonance imaging. J Clin Oncol 21: 1094-1100
88. Itoh R, Melhem ER, Mori S, Eichler FS, Raymond GV, Moser HW (2001) Diffusion tensor brain MR imaging in $\mathrm{X}$-linked cerebral adrenoleukodystrophy. Neurology 56: 544-547

89. Eichler F, Itoh R, Barker PB, (2003) Proton MR spectroscopic and diffusion tensor brain MR imaging in X-linked adrenoleukodystrophy: initial experience. Radiology 225: 245-252

90. Schneider JFL, Il'yasov KA, Boltshauser E, Hennig J, Martin E (2003) Diffusion tensor imaging in cases of adrenoleukodystrophy: preliminary experience as a marker for early demyelination? ANJR 24: 819-824

91. Guo AC, Petrella JR, Kurtzberg J, Provenzale JM (2001) Evaluation of white matter anisotropy in Krabbe disease with diffusion tensor imaging: initial experience. Radiology 218: 245-252

92. Ono J, Harada K, Takahashi M, et al (1995) Differentiation between dysmyelination and demyelination using magnetic resonance diffusional anisotropy. Brain Res 671: 141-148
93. Ono J, Harada K, Mano T, Sakurai K, Okada S (1997) Differentiation of dysand demyelination using diffusional anisotropy. Pediatr Neurol 16: 63-66

94. Schaumburg H, Powers J, Raine C, Suzuki K, Richardson E (1975) Adrenoleukodystrophy: a clinical and pathological study of 17 cases. Arch Neurol 32: 577-591

95. Ellis CM, Simmons A, Jones DK, (1999) Diffusion tensor MRI assesses corticospinal tract damage in ALS. Neurology 53: 1051-1058

96. Chabriat H, Pappata S, Poupon C, et al (1999) Diffusion tensor imaging in cerebral autosomal dominant arteriopathy with subcortical infarcts and leukoencephalopathy (CADASIL): preliminary results [abstract] Stroke 30: P62

97. Chabriat H, Pappata S, Poupon C, et al (1999) Clinical severity in CADASIL related to ultrastructural damage to white matter - in vivo study with diffusion tensor MRI. Stroke 30: 2637-2643

98. Filippi CG, Ulug AM, Ryan E, Ferrando SI, van Gorp W (2001) Diffusion tensor imaging in patients with HIV and normal-appearing white matter on MR images of the brain. AJNR 22: 277-283 